\title{
Correlation of school absenteeism and laboratory results for Flu A in Alberta, Canada
}

\author{
Elizabeth Birk-Urovitz ${ }^{* 2,3}, \mathrm{Ye} \mathrm{Li}^{1,2}$, Steven Drews ${ }^{4,5}$, Christopher Sikora ${ }^{4,5}$, \\ Deena Hinshaw ${ }^{4,5}$, Rita K. Biel ${ }^{4}$, Faiza Habib ${ }^{4}$, Laura Rivera ${ }^{6}$, Hussain Usman ${ }^{4}$, \\ David Strong ${ }^{4,6}$ and lan Johnson ${ }^{1,2}$
}

${ }^{1}$ Public Health Ontario, Toronto, ON, Canada; '2University of Toronto, Toronto, ON, Canada; ${ }^{3}$ UC Berkeley, Berkeley, CA, USA; ${ }^{4}$ Alberta Health Services, Edmonton, AB, Canada; ${ }^{5}$ University of Alberta, Edmonton, AB, Canada; ${ }^{6}$ University of Calgary, Calgary, AB, Canada

\section{Objective}

To assess the correlations between weekly rates of elementary school absenteeism due to illness (SAi) and percent positivity for influenza A from laboratory testing (PPFluA) when conducted at a city level from September to December over multiple years.

\section{Introduction}

Rates of student absenteeism in schools have been mainly used to detect outbreaks in schools and prompt public health action to stop local transmission ${ }^{1,2}$. A report by Kim Mogto et al. ${ }^{3}$ stated that aggregated counts of school absenteeism (SAi) were correlated with PPFluA, but the sample may have been biased. The purpose of this study was to assess the correlation between aggregated rates of SAi and PPFluA for two cities, Calgary and Edmonton, in Alberta. In such situations, SAi could potentially be used as a proxy for PPFluA when there are not enough samples for stable laboratory estimates.

\section{Methods}

The Alberta Real-Time Syndromic Surveillance Net (ARTSSN) ${ }^{4}$ collects elementary SA data from the two major school boards in two cities in Alberta with populations $>800,000$. Since reasons for SA are stated, rates of SAi can be calculated. Data were obtained for three years, 2012 to 2014, for each city. Laboratory data on tests of respiratory agents using a standardized protocol were obtained from Alberta's Provincial Laboratory for Public Health for the same time period and locations. The dates of the specimens being received by the laboratory were used in this analysis. For each data source, the relative proportions (SAi and PPFluA) were calculated. Data for the first week of school in September and for the last two weeks of December were removed for each year due to the SAi rates being unstable. Linear regression models were constructed, with rates of SAi predicted by PPFluA. Separate models were run for each city and for each year, resulting in a total of 6 models. Percent positivity for entero-rhinoviruses (PPERV) was added to see if it improved the models. The regression models were created using Excel and checked in the statistical programs, SAS and R. An analysis to assess the influence of a lag period was assessed using $\mathrm{R}$.

\section{Results}

For each city, the provincial lab tested between 4,000 and 6,000 specimens each fall and SAi rates were based on denominators of between 20,000 and 36,000 children. The $\mathrm{R}^{2}$, betas, and p-values for all 6 regression models are shown in Table 1 . The minimum correlation value was 0.693 and the maximum was 0.935 . Due to the strong negative correlations between PPERV and PPFluA, PPERV was not retained in the models. Looking at the lag periods, the maximum correlations occurred at a zero week lag in two years (2012 and 2014) and at a -1 week lag in 2013. The two years with a zero lag were both dominated by a H3N2 strain while the year with mainly a H1N1 strain showed a lag of -1 . Only one year of H1N1 data was available for analysis.

\section{Conclusions}

We observed strong correlations between the weekly rates of elementary SAi and PPFluA at the city level over three years, from September to December. The reasons for the difference in lag times between the H1N1 and H3N2 seasons are being investigated.

Table 1: Results of the Linear Regression Models predicting SAi by PPFluA

\begin{tabular}{|c|c|c|c|c|}
\hline City & Fall of the Year & R-Squared (Flu A only) & Correlation Coefficient & Slope (Beta) for Flu A \\
\hline Edmonton & 2012 & 0.82 & 0.905 & $0.952^{*}$ \\
\hline Edmonton & 2013 & 0.48 & 0.693 & $0.449^{* *}$ \\
\hline Edmonton & 2014 & 0.86 & 0.927 & $0.405^{*}$ \\
\hline Calgary & 2012 & 0.82 & 0.906 & $0.816^{*}$ \\
\hline Calgary & 2013 & 0.63 & 0.794 & $0.406^{*}$ \\
\hline Calgary & 2014 & 0.88 & 0.935 & $0.524^{*}$ \\
\hline
\end{tabular}

${ }^{*} \mathrm{p}<0.001,{ }^{* *} \mathrm{p}<0.01$

\section{Keywords}

School absenteeism; Influenza A; Correlation; Syndromic Surveillance

\section{References}

1. Mook P, Joseph C, Gates P, Phin N. Pilot scheme for monitoring sickness absence in schools during the 2006/07 winter in England: can these data be used as a proxy for influenza activity?. European Communicable Disease Bulletin. 2007 Dec;12(12):E11-2.

2. Besculides M, Heffernan R, Mostashari F, Weiss D. Evaluation of school absenteeism data for early outbreak detection, New York City. BMC Public Health. 2005;5:105.

3. Kom Mogto CA, De Serres G, Douville Fradet M, Lebel G, Toutant $\mathrm{S}$, Gilca R, et al. School absenteeism as an adjunct surveillance indicator: experience during the second wave of the 2009 H1N1 pandemic in Quebec, Canada. PLoS ONE [Electronic Resource] 2012;7(3):e34084.

4. Fan S, Blair C, Brown A, Gabos S, Honish L, Hughes T, et al. A multifunction public health surveillance system and the lessons learned in its development: the Alberta Real Time Syndromic Surveillance Net. Can J Public Health. 2010;101(6):454-8.

\section{*Elizabeth Birk-Urovitz}

E-mail: elizabeth.birk.urovitz@mail.utoronto.ca 